\title{
Postpartum depression in mothers and fathers: a structural equation model
}

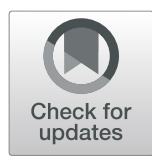

Zhizhou Duan ${ }^{1,2 \dagger}$, Yuanyuan Wang ${ }^{3,4 \dagger}$, Ping Jiang ${ }^{1 \dagger}$, Amanda Wilson ${ }^{4 \dagger}$, Yan Guo ${ }^{1}$, Yongliang LV ${ }^{1}$, Xiaonan Yang ${ }^{1}$, Renjie Yu', Shuilan Wang ${ }^{1}$, Zhengyan Wu', Mengqing Xia' ${ }^{1}$, Guosheng Wang ${ }^{1}$, Ye Tao ${ }^{5}$, L Xiaohong ${ }^{1}$, Ling Ma ${ }^{5}$, Hong Shen ${ }^{5}$, Jue Sun ${ }^{6}$, Wei Deng ${ }^{7}$, Yong Yang ${ }^{1 *}$ and Runsen Chen ${ }^{3}$

\begin{abstract}
Background: Post-partum depression (PPD) is a growing mental health concern worldwide. There is little evidence in the Chinese context of the relationship between paternal PPD and maternal PPD. Given the growing global concerns this relationship requires further exploration.

Methods: A survey was conducted with 950 total couples from March 2017 to December 2018. The study was conducted using a standardized questionnaire that included basic demographic information, information on the relationship between the mother-in-law and daughter-in-law, marital satisfaction (both maternal and paternal), and PPD symptoms. Structural Equation Modelling (SEM) analysis was used to explore the underlying mechanism for PPD symptoms in mothers and fathers.

Results: In $4.4 \%$ of the couples both the wife and the husband showed depressive symptoms. Maternal marital satisfaction showed a significant mediating effect on paternal PPD $(B=-0.114, p<0.01)$, and there was a direct effect of maternal PPD on paternal PPD $(B=0.31, p<0.001)$.

Conclusions: This is the first study to investigate the possible correlation between maternal PPD, mother-in-law and daughter-in-law relationship satisfaction, maternal marital satisfaction, paternal marital satisfaction, and paternal PPD. It is important for future PPD interventions to target both maternal and paternal mental health, as well as the mechanisms identified that can lead to PPD.
\end{abstract}

Keywords: paternal post-partum depression, maternal post-partum depression, mother-in-law relationship, marital satisfaction, Sturctural Equation Modelling (SEM)

\section{Background}

Post-partum depression (PPD) is a growing mental health concern and one of the leading causes of poor familial and infant health outcomes [1-3]. Similar to maternal PPD, PPD can also effect the mental health of fathers [4]. Paternal PPD refers to a new father who suffers from depressive symptoms

\footnotetext{
* Correspondence: szgjyy@126.com

${ }^{\dagger}$ Zhizhou Duan, Yuyuan Wang, Ping Jiang and Amanda Wilson contributed equally to this work.

'Institute of Mental Health, Suzhou Guangji Hospital, Affiliated Guangji Hospital of Soochow University, Soochow University, No. 11 Guangqian Road, Jiangsu Province 215137 Suzhou, PR China

Full list of author information is available at the end of the article
}

within a 12 month perinatal period after the birth of an infant $[5,6]$. Paternal PPD has a negative impact on family members, the impact can reduce early parent-infant interactions resulting in poor communication and stimulation [7].It can also have further long-term adverse impacts on an infant's physical growth and their cognitive, behavioral, and social development $[8,9]$. Previous research has shown the rate of paternal PPD ranges from 5.4-13.6\%, depending on sample size, measurement scales, and different population characteristics [4, 10-12]. The high rate of paternal PPD has received worldwide concern [4], and research should be conducted to provide an evidence base to this timely subject. 
To date, several risk factors of paternal PPD have been identified including maternal PPD, marital satisfaction, and social support [11, 13-15]. The state of a new mother's perinatal mental health has been found to influence paternal PPD $[14,16]$. The rate of paternal PPD is also significantly associated with rates of maternal PPD $[4,17]$.If there is maternal PPD this has been found to be the strongest direct predictor of paternal PPD, which may be explained by the couple's adjustment within a new stage of parenthood where changes in factors of partner support and relationship satisfaction can impact on the mood of both new mothers and fathers, increasing their risk for depression [16].

Paternal PPD has also been strongly associated with paternal marital satisfaction. A previous study has indicated that marital satisfaction was one of the most important predictors for paternal PPD [4]. According to a longitudinal study surveyed in Hong Kong, an inharmonious marriage can increase the risks of paternal PPD [18]. In addition, previous research has found that the mother-in-law relationship can largely decrease the level of paternal marital satisfaction [19]. According to a study in China, maternal marital satisfaction also has a large impact on paternal marital satisfaction, meaning those with higher maternal marital satisfaction are more likely to have greater levels of paternal marital satisfaction [20].

In addition, a good relationship between the mother-inlaw and daughter-in-law is positively correlated with a higher degree of maternal marital satisfaction [21]. And in turn, the women experiencing postpartum depression can aggravate the relationship with the mother-in-law, negatively impacting marital satisfaction further [18]. Within Chinese culture, the relationship with the mother-in-law and maternal marital satisfaction are closely linked to maternal $\operatorname{PPD}[2,18,22]$. A poor relationship with the mother-in-law and maternal marital satisfaction can therefore significantly increase the risk of maternal PPD [2].

Unlike maternal PPD, there are insufficient studies focused on PPD among new fathers to further understand the factors and associations of paternal PPD. Therefore, we aimed to use structural equation modeling (SEM) to explore the relationship among maternal PPD, mother-inlaw relationship satisfaction, maternal marital satisfaction, paternal marital satisfaction, and paternal PPD. We hypothesized that (1) maternal marital satisfaction and mother-in-law relationship satisfaction could indirectly impact on paternal PPD via paternal marital satisfaction; (2) maternal PPD, mother-in-law relationship satisfaction, and maternal marital satisfaction would be associated.

\section{Methods}

\section{Participants}

This cross-sectional study was conducted in 3 local community health service centers and obstetric wards in Suzhou city, China between March 2017 and December 2018 [23-25].
Participants who met the following inclusion criteria were included in the study: (1) the participants were married; (2) participants were equal or above the legal age of marriage (20 years old for women, and 22 years old for men) [26]; (3) women who have given birth in the last year; (4) both partners in the couples agreed to participate and provided informed consent. A total of 1,034 couples were recruited and 950 couples met the inclusion criteria. All data was collected by trained researchers. Following the Declaration of Helsinki, the researchers fully illustrated the purpose of the study in the information sheet, considerations were made to protect the privacy of participants through assigning them a participant number, and the information sheet informed participants of their right to withdraw at any point, including how to do so [27]. The Ethics Committee of the Suzhou Guangji Hospital in China (reference number: 2016-010) approved this study.

\section{Measures \\ Basic Socio-demographic characteristics}

Basic Socio-demographic characteristics were collected including age, residence, whether the new father was the only child, yearly family income, educational level, and if there was any family history of psychological disorders.

\section{Mother-in-law relationship}

The relationship between mother-in-law and daughterin-law was assessed using the question "Do you currently feel satisfied with the relationship with your mother-in-law?" (answered by the wife). The options on the likert scale ranged from 1 (extremely unsatisfactory) to 7 (extremely satisfactory). This question has been used in previous research to measure satisfaction of the wife within the mother-in-law relationship [2].

\section{Marital Satisfaction (wife/husband)}

Marital satisfaction (wife/husband) was measured by the question "Do you currently feel satisfied in your marital relationship?" (answered by both the wife and husband). The participants could respond on a scale of extremely unsatisfactory (scored 1) to extremely satisfactory (scored 7). This question has been used for marital satisfaction assessment in previous research [28].

\section{Postpartum depression (wife/husband)}

For both wives and husbands, PPD was measured by the Edinburgh Postnatal Depression Scale (EPDS). The EPDS is a 10-item 4-point Likert scale, with a higher total score indicating a higher risk of PPD. The cut-off score of the EPDS scale was set to 10 to indicate depressive signs [29]. A Chinese version of the EPDS was used (the Cronbach alpha ranged from 0.79 to 0.87 ) [30, 31]. In this study, the Cronbach alpha was 0.85 (wife) and 0.83 (husband) respectively. 


\section{Statistical analysis}

For the basic socio-demographic characteristics, we used frequencies and percentages to summarize related characteristics. A Spearman's Correlation analysis was calculated to explore the relationship between the variables. The above analysis used SPSS (version 21.0) and the significance level was considered at $p<0.05$ (twotailed). For the SEM analysis, we used SPSS AMOS module 20.0 to test our hypothesized model. The SEM model was applied to understand how several paths when combined could predict how maternal marital satisfaction and mother-in-law relationship satisfaction indirectly impact on paternal PPD via paternal marital satisfaction. It also looked at the association between maternal PPD, mother-in-law relationship satisfaction, and maternal marital satisfaction. The SEM model was a good fit, with indexes as follows: Comparative fit index, CFI $>0.90$; Tucker Lewis index, TLI $>0.90$, Goodness of fit index, GFI $>0.90$; Normed fit index, NFI $>0.90$; and had a root mean square error of approximation, RMSEA $<0.05$ and $\mathrm{x} 2 / \mathrm{df}<3(P>0.05)$ $[32,33]$. In the above indicies CFI, $\chi^{2} / \mathrm{df}$, and absolute fit directly assess how well a model fits the observed data; RMSEA, corrects for a model's complexity and other indices compensate for the effect of model complexity [33].

\section{Results}

As shown in Table 1, among the total 950 couples eligible to participate, the mean age of a new father was 31.7 years old $(S D=3.93)$ and $52.9 \%$ of new fathers were between 31 and 35 years old. Among all new father participants, $55.3 \%$ were urban residence and $45.4 \%$ were the only child. More than half of the participants (64.7\%) reported a yearly family income of more than 15 (unit in ten thousand yuan) and most of the individuals (81.2\%) had a bachelor's degree or higher. Also, $4.3 \%$ of individuals had a family history of psychological disorders, $10.2 \%$ showed depressive symptoms, and in $4.4 \%$ of the couples both the wife and husband showed depressive symptoms.

In Table 2, the correlation analysis shows that paternal PPD was positively correlated with maternal PPD $(r=$ $0.423, p<0.01)$ and negatively correlated with motherin-law relationship satisfaction $(r=-0.141, p<0.01)$, maternal marital satisfaction $(r=-0.193, p<0.01)$, and paternal marital satisfaction $(r=-0.157, p<0.01)$. Suggesting those with higher levels of maternal PPD, lower levels of mother-in-law relationship satisfaction, and lower levels of marital satisfaction are more likely to be correlated with having higher levels of paternal PPD. Furthermore, the five key variables were significantly correlated with each other $(p<0.01)$.
Table 1 Socio-demographic characteristics of new fathers

\begin{tabular}{|c|c|c|}
\hline Characteristic & Number & Percent(\%) \\
\hline \multicolumn{3}{|l|}{ Age } \\
\hline $22-25$ & 33 & 3.6 \\
\hline $26-30$ & 251 & 27.6 \\
\hline $31-35$ & 481 & 52.9 \\
\hline$\geq 36$ & 145 & 15.9 \\
\hline Mean, SD & 31.67 & 3.93 \\
\hline \multicolumn{3}{|l|}{ Residence } \\
\hline Rural & 425 & 44.7 \\
\hline Urban & 525 & 55.3 \\
\hline \multicolumn{3}{|l|}{ Being the only child } \\
\hline Yes & 431 & 45.4 \\
\hline No & 519 & 54.6 \\
\hline \multicolumn{3}{|c|}{ Yearly family income (ten thousand yuan) } \\
\hline$<15$ & 335 & 35.3 \\
\hline $15-30$ & 483 & 50.9 \\
\hline$>30$ & 131 & 13.8 \\
\hline \multicolumn{3}{|l|}{ Educational level } \\
\hline High school or lower & 179 & 18.8 \\
\hline Bachelor degree or higher & 771 & 81.2 \\
\hline \multicolumn{3}{|l|}{ Marital status } \\
\hline First marriage & 946 & 99.6 \\
\hline Remarriage & 4 & 0.4 \\
\hline \multicolumn{3}{|c|}{ Family mental health history } \\
\hline Yes & 41 & 4.3 \\
\hline No & 909 & 95.7 \\
\hline \multicolumn{3}{|c|}{ Depression symptom (new father) } \\
\hline$\geq 10$ & 97 & 10.2 \\
\hline$<10$ & 853 & 89.8 \\
\hline \multicolumn{3}{|c|}{ Depression symptom (couples) } \\
\hline$\geq 10$ & 42 & 4.4 \\
\hline$<10$ & 908 & 95.6 \\
\hline
\end{tabular}

In Fig. 1, the final SEM model shows the mechanism of maternal PPD, mother-in-law relationship satisfaction, maternal marital satisfaction, paternal marital satisfaction, and paternal PPD. The final SEM model indicated a good fit $\left(x^{2} / d f=2.443, p=0.118, \quad R M S E A=0.039\right.$, $\mathrm{GFI}=0.999, \mathrm{CFI}=0.998, \mathrm{NFI}=0.997, \mathrm{TLI}=0.982$ ). Fur thermore, the final model revealed that the specific standardized effect of maternal PPD on paternal PPD was $0.31(\mathrm{~B}=0.31, p<0.001)$ showing that paternal marital satisfaction predicted paternal $\mathrm{PPD}, \mathrm{B}=-0.41, p<0.001$, and maternal marital satisfaction predicted paternal marital satisfaction, $\mathrm{B}=-0.41, p<0.001$. In addition, maternal marital satisfaction, mother-in-law relationship, 
Table 2 Key variables and Spearman's Correlation coefficients

\begin{tabular}{|c|c|c|c|c|c|c|c|}
\hline & Mean & SD & 1 & 2 & 3 & 4 & 5 \\
\hline 1. Mother-in-law relationship & 5.40 & 1.30 & 1 & & & & \\
\hline 2. Maternal marital satisfaction & 5.67 & 1.19 & $0.728^{* *}$ & 1 & & & \\
\hline 3. Maternal postpartum depression & 5.83 & 4.80 & $-0.168^{* *}$ & $-0.188^{* *}$ & 1 & & \\
\hline 4. Paternal marital satisfaction & 6.14 & 0.95 & $0.307^{* *}$ & $0.423^{* *}$ & $-0.160^{* *}$ & 1 & \\
\hline 5. Paternal postpartum depression & 4.07 & 3.99 & $-0.141^{* *}$ & $-0.193^{* *}$ & $0.423 * *$ & $-0.157^{* *}$ & 1 \\
\hline
\end{tabular}

and maternal PPD were significantly correlated with each other in the model (maternal marital satisfaction and mother-in-law relationship, $\mathrm{B}=0.99, \quad p<0.001$; mother-in-law relationship and maternal PPD, $\mathrm{B}=-1.07$, $p<0.001$; maternal marital satisfaction and maternal PPD, $\mathrm{B}=-1.06, p<0.001)$. All in all, the total effect of maternal marital satisfaction on paternal PPD was $\mathrm{B}=$ $-0.11(p<0.01)$, and maternal marital satisfaction had an indirect effect on paternal PPD by paternal marital satisfaction. The effect of mother-in-law relationship satisfaction on paternal marital satisfaction was not significant.

\section{Discussion}

To our knowledge, this is the first study to explore the possible correlations between maternal PPD, mother-in-law relationship satisfaction, maternal marital satisfaction, paternal marital satisfaction, and paternal PPD using SEM analysis. Our research confirms previous findings in which maternal PPD plays an important role in predicting paternal PPD. In our study, our outcomes showed the prevalence of paternal PPD in the couples sampled. This rate is consistent with previous research, with a recent meta-analysis reporting similar overall rates of PPD in new fathers during the initial perinatal period [4]. Targeted towards new fathers in China, another recent meta-analysis also showed a prevalence of paternal PPD that was consistent with our study [34].

The model further showed that maternal marital satisfaction had an indirect effect on paternal PPD by paternal marital satisfaction. Therefore, our study suggests that due attention should been given to a new mothers' condition and that signs of marital dissatisfaction should also be considered as a risk factor for paternal PPD. Screening new mothers for PPD and martial satisfaction would then create more effective interventions to identify if there is a need to screen new fathers for PPD, providing a window for early intervention for paternal PPD and familial and infant health [35]. Paternal marital satisfaction was a full mediating factor in the relationship between maternal marital satisfaction and paternal PPD. Although there is a lack of research that reports how paternal marital satisfaction can predict the development of paternal PPD, it has been supported in the previous literature, indirectly indicating that, a disharmonious marriage can further increase the risk of paternal PPD, and that furthermore maternal marital satisfaction can exert considerable influence on paternal marital satisfaction $[20,36]$. As a result, our study suggests that both maternal and paternal marital satisfaction be taken into consideration as another risk factor to consider when

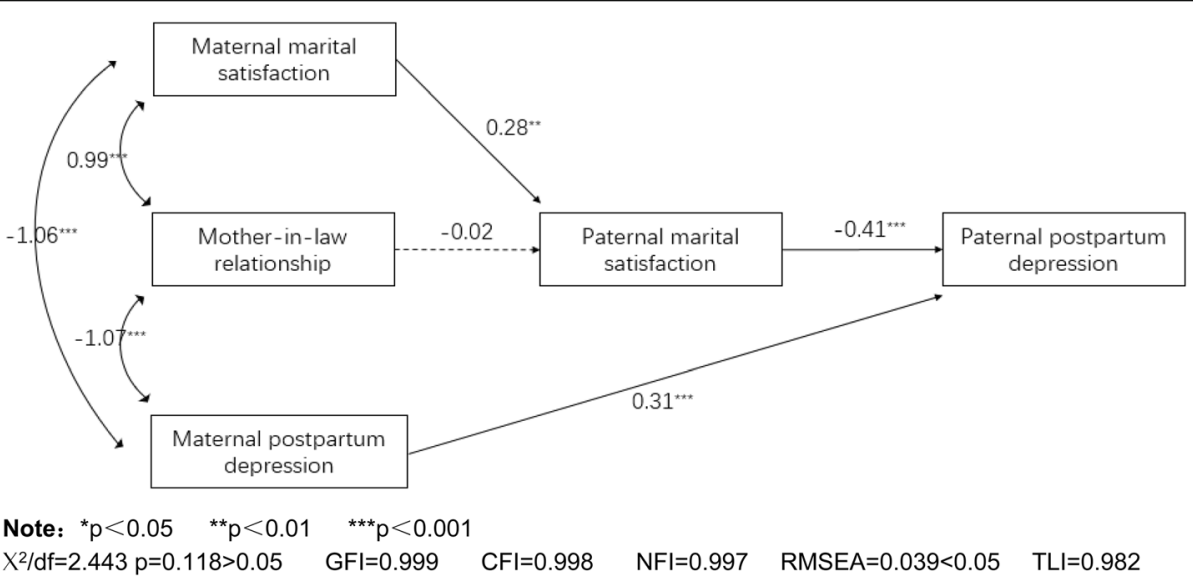

Fig. 1 The final mediation model. Note: ${ }^{*} p<0.05{ }^{* *} p<0.01{ }^{* *} p<0.001 . \mathrm{X} 2 / \mathrm{df}=2.443 \mathrm{p}=0.118>0.05 \mathrm{GF}=0.999 \mathrm{CF}=0.998 \mathrm{NFI}=0.997$ RMSEA $=0.039<0.05 \mathrm{TL}=0.982$ 
aiming to reduce the rates of paternal PPD. If the new mother shows both signs of PPD and martial dissatisfaction, this study recommends screening of fathers not only for PPD, but also for marital dissatisfaction as well. The ability to identify paternal PPD and marital dissatisfaction earlier would have a positive impact on the child's lifespan development, amongst other key cognitive, behavioral, and social aspects, as suggested in the literature reviewed.

In this study the mother-in-law relationship was not associated with paternal marital satisfaction, which is in contrast to other research [19]. However, the previous studies do not consider the effects of maternal marital satisfaction in conjunction with paternal marital satisfaction. Therefore, there should be caution around the assumption that a poor relationship with the mother-inlaw can predicted the level of paternal marital satisfaction directly. However, further research is needed that looks at the maternal and paternal mother-in-law relationships to provide a more robust base of evidence. In our current study, mother-in-law relationship satisfaction should still receive due attention because of the potentially effect on maternal marital satisfaction and maternal postpartum depression, even though it indirectly predicted the level of paternal marital satisfaction. If a new mother is dissatisfied with the mother-in-law relationship, her dissatisfaction likely could lead to marital dissatisfaction, which this study further shows predicts paternal PPD and marital dissatisfaction.

In summary, our study indicated that the three key factors related to the wife (maternal PPD, mother-in-law relationship satisfaction, and maternal marital satisfaction) were significantly associated. These three factors can act on paternal PPD by the pathway of paternal marital satisfaction. One of the most common familial conflicts that occurs is within the relationship between the wife and mother-in-law in China, which is a key aspect of Chinese culture [2]. It can increase the family disharmony and have an important impact on marital satisfaction between a wife and husband [22], increasing the risk of paternal PPD. Therefore, more attention should focus on the family network and the relationship among family members, with the goal of forming a harmonious atmosphere within the family. Interventions should then target the whole family if there is maternal dissatisfaction with the mother-in-law relationship. This disharmony should be identified prior or during pregnancy to buffer against maternal and paternal PPD. This could be achieved by using the scales mentioned in this study during prenatal appointments.

There are some limitations to the present study. First, this study was a cross-sectional survey in one city in China, which deceases the levels of representativeness of the sample and a causal relationship cannot be fully established. Second, some of the factors influencing how women can increase the risk of paternal PPD were not surveyed due to the accessibility of couples. Third, the measure of the history of family psychological disorders and marital satisfaction were self-reported, which may cause some bias in this study. While we have tried to demonstrate the relationship between maternal PPD and paternal PPD on marital satisfaction, future studies should explore paternal PPD and other related risk factors to create more effective intervention measures.

\section{Conclusions}

In conclusion, our study provided that the mechanism of paternal PPD that best predicted a new mother's marital satisfaction and risk of PPD accordingly. Maternal PPD and maternal marital satisfaction were found to be central factors when reducing the prevalence of paternal PPD. Therefore, there should be considerable importance placed on seeking to improve marital satisfaction and the mother-in-law relationship satisfaction amongst the mother-in-law and the wife.

\section{Abbreviations}

PPD: Postpartum depression; SEM: Structural equation model (SEM); EPDS: Edinburgh Postnatal Depression Scale

\section{Acknowledgements}

We thank all the participants and researchers who made contribution to the study.

\section{Authors' contributions}

Study design: YY, DW, SJ; Study implementation and data collection: JP, GY, LY, YX, YR, WS, WZ, XM, WG, TY, LX, ML, HS; Data analysis: YY, DZ, WY; Data interpretation $Y Y, C R, D Z, W Y, A W$, JP; Manuscript preparation: $Y Y, C R, D Z$, $W Y, A W, J P ; A l l$ authors read and approved the final manuscript.

\section{Funding}

The study was supported Young Medical Talent of Jiangsu Province (QNRC2016229), Suzhou Key Diagnosis and Treatment Program (LCZX201616), Suzhou Key Medical Center for Psychiatric Diseases (SZZX201509), Suzhou Municipal Sci-Tech Bureau Program (SYSD2012125), Health City "531" action plan of Suzhou Municipal and Suzhou Clinical Medicine Expert Team (SZYJTD201715). The funders had no role in study design, data collection, data analysis, data interpretation and writing of the manuscript.

\section{Availability of data and materials}

The datasets used and/or analysed during the current study are available from the corresponding author on reasonable request.

\section{Ethics approval and consent to participate}

This study received approval by the Human Research and Ethics Committee of Suzhou Guangji Hospital, China (Ref:2016-010). The written informed consent was obtained from the participants.

\section{Competing interests \\ None.}

\section{Author details}

${ }^{1}$ Institute of Mental Health, Suzhou Guangji Hospital, Affiliated Guangji Hospital of Soochow University, Soochow University, No. 11 Guangqian Road, Jiangsu Province 215137 Suzhou, PR China. ${ }^{2}$ School of Health Sciences, Wuhan university, 430072 Wuhan, Hubei Province, China. ${ }^{3}$ National Clinical Research Center for Mental Disorders, Department of Psychiatry, and China National Technology Institute on Mental Disorders, The Second Xiangya Hospital of Central South University, 410011 Changsha, Hunan, China. 
${ }^{4}$ Division of Psychology, Faculty of Health and Life Sciences, De Montfort University, Leicester, UK. ${ }^{5}$ Suzhou science \& technology town hospital, Suzhou, China. ${ }^{6}$ Gusu District Wumenqiao Street Canglang Xincheng Community Health Service Center, Suzhou, China. ${ }^{7}$ Mental Health Center, West China Hospital of Sichuan University, Chengdu, PR China.

Received: 8 October 2019 Accepted: 1 September 2020

Published online: 15 September 2020

\section{References}

1. O'Hara MW, McCabe JE. Postpartum depression: current status and future directions. Ann Rev Clin Psychol. 2013;9:379-407.

2. Xiong R, Deng A, Wan B, Liu Y. Prevalence and factors associated with postpartum depression in women from single-child families. Int J Gynaecol Obstet. 2018;141(2):194-9.

3. Beardslee WR, Versage EM, Gladstone TR. Children of Affectively III Parents: A Review of the Past 10 Years. Journal of the American Academy of Child Adolescent Psychiatry. 1998;37(11):1134-41.

4. Paulson JF, Bazemore SD. Prenatal and postpartum depression in fathers and its association with maternal depression: a meta-analysis. Jama. 2010; 303(19):1961-9.

5. Musser AK, Ahmed AH, Foli KJ, Coddington JA. Paternal Postpartum Depression: What Health Care Providers Should Know. Journal of Pediatric Health Care. 2013:27(6):479-85.

6. Brockington I. Postpartum psychiatric disorders. Lancet. 2004;363(9405):303-10.

7. Murray L, Halligan S. Postnatal depression and young children's development. 2018. p. 172-86.

8. Ramchandani P, Stein A, Evans J, O'Connor TG. Paternal depression in the postnatal period and child development: a prospective population study. The Lancet. 2005;365(9478):2201-5.

9. Perez CF, Brahm MP. [Paternal postpartum depression: Why is it also important?]. Revista chilena de pediatria. 2017;88(5):582-5.

10. Edward K, Castle D, Mills C, Davis L, Casey J. An Integrative Review of Paternal Depression. American Journal of Men's Health. 2014;9(1):26-34.

11. Anding JE, Rohrle B, Grieshop M, Schucking B, Christiansen H. Couple comorbidity and correlates of postnatal depressive symptoms in mothers and fathers in the first two weeks following delivery. J Affect Disord. 2016; 190:300-9.

12. Nishimura A, Fujita Y, Katsuta M, Ishihara A, Ohashi K. Paternal postnatal depression in Japan: an investigation of correlated factors including relationship with a partner. BMC Pregnancy Childbirth. 2015;15:128.

13. Bergstrom M. Depressive symptoms in new first-time fathers: associations with age, sociodemographic characteristics, and antenatal psychological well-being. Birth (Berkeley, Calif). 2013;40(1):32-8.

14. Serhan N, Ege E, Ayranci U, Kosgeroglu N. Prevalence of postpartum depression in mothers and fathers and its correlates. Journal of clinical nursing. 2013;22(1-2):279-84.

15. Da Costa D, Zelkowitz P, Dasqupta K, Sewitch M, Lowensteyn I, Cruz R Hennegan K, Khalife S. Dads Get Sad Too: Depressive Symptoms and Associated Factors in Expectant First-Time Fathers. Am J Mens Health. 2017; 11(5):1376-84

16. Goodman JH. Paternal postpartum depression, its relationship to maternal postpartum depression, and implications for family health. Journal of advanced nursing. 2004;45(1):26-35

17. Pinheiro RT, Magalhaes PV, Horta BL, Pinheiro KA, da Silva RA, Pinto RH. Is paternal postpartum depression associated with maternal postpartum depression? Population-based study in Brazil. Acta psychiatrica Scandinavica. 2006;113(3):230-2.

18. Koh YW, Lee AM, Chan CY, Fong DY, Lee CP, Leung KY, Tang CS. Survey on examining prevalence of paternal anxiety and its risk factors in perinatal period in Hong Kong: a longitudinal study. BMC Public Health. 2015;15:1131-45.

19. Wang YY, Li H, Wang YJ, Wang H, Zhang YR, Gong L, Ma J, Wang Y, Wang MZ, Qiu SX, et al. Living with parents or with parents-in-law and postpartum depression: A preliminary investigation in China. J Affect Disord. 2017;218:335-8.

20. Cao H, Yuan X, Fine M, Zhou N, Fang X. Neuroticism and Marital Satisfaction During the Early Years of Chinese Marriage: The Mediating Roles of Marital Attribution and Aggression. Family process. 2019;58(2):478-95

21. Shih KY, Pyke K. Power, Resistance, and Emotional Economies in Women's Relationships With Mothers-in-Law in Chinese Immigrant Families. J Fam Issues. 2010;31(3):333-57.
22. Top ED, Cetisli NE, Guclu S, Zengin EB. Paternal Depression Rates in Prenatal and Postpartum Periods and Affecting Factors. Arch Psychiatr Nurs. 2016; 30(6):747-52.

23. Duan Z, Wang Y, Tao Y, Bower JL, Yu R, Wang S, Wu Z, Lv Y, Yang X, Li X. Relationship between trait neuroticism and suicidal ideation among postpartum women in China: Testing a mediation model. J Affect Disord. 2019;256:532-5

24. Lu L, Duan Z, Wang Y, Wilson A, Yang Y, Zhu L, Guo Y, Lv Y, Yang X, Yu R. Mental health outcomes among Chinese prenatal and postpartum women after the implementation of universal two-child policy. J Affect Disord. 2020; 264:187-92.

25. Wang Y, Li X, Duan Z, Wilkinson MR, Yu R, Li S, Huang L, Guo Y, Ma L, Dong Q. Screening for postpartum hypomania among Chinese women after childbirth. Psychiatry Research-neuroimaging. 2019;282:112591-1.

26. Hare-Mustin RT. China's Marriage Law: a model for family responsibilities and relationships. Family process. 1982;21(4):477-81.

27. Association WM. Declaration of Helsinki: ethical principles for medical research involving human subjects. 2008.

28. Ip P, Li TMH, Chan KL, Ting AYY, Chan CY, Koh YW, Ho FKW, Lee A. Associations of paternal postpartum depressive symptoms and infant development in a Chinese longitudinal study. Infant Behav Dev. 2018:53:81-9.

29. Matthey S, Barnett B, Kavanagh DJ, Howie P. Validation of the Edinburgh Postnatal Depression Scale for men, and comparison of item endorsement with their partners. J Affect Disord. 2001:64(2-3):175-84.

30. Wang Y, Guo X, Lau Y, Chan KS, Yin L, Chen J. Psychometric evaluation of the Mainland Chinese version of the Edinburgh Postnatal Depression Scale. International journal of nursing studies. 2009;46(6):813-23.

31. Jia L, Ji FY, Wu J, Wang Y, Wu CQ. Paternal depressive symptoms during the early postpartum period and the associated factors following the implementation of the two-child policy in China. Arch Psychiatr Nurs. 2020; 34(2):43-9.

32. Hu Lt, Bentler PM. Cutoff criteria for fit indexes in covariance structure analysis: Conventional criteria versus new alternatives. Struct Equ Model. 1999;6(1):1-55.

33. Weston R, Gore PA. A Brief Guide to Structural Equation Modeling. The Counseling Psychologist. 2006;34(5):719-51.

34. Wang T, Xu Y, Li Z, Chen L. [Prevalence of paternal postpartum depression in China and its association with maternal postpartum depression: A Metaanalysis]. Zhong nan da xue xue bao Yi xue ban = Journal of Central South University Medical sciences. 2016;41(10):1082-9.

35. Dudley M, Roy K, Kelk N, Bernard D. Psychological correlates of depression in fathers and mothers in the first postnatal year. Journal of Reproductive Infant Psychology. 2001;19(3):187-202.

36. Gawlik S, Muller M, Hoffmann L, Dienes A, Wallwiener M, Sohn C, Schlehe B, Reck $C$. Prevalence of paternal perinatal depressiveness and its link to partnership satisfaction and birth concerns. Arch Women Ment Health. 2014;17(1):49-56.

\section{Publisher's Note}

Springer Nature remains neutral with regard to jurisdictional claims in published maps and institutional affiliations.

Ready to submit your research? Choose BMC and benefit from

- fast, convenient online submission

- thorough peer review by experienced researchers in your field

- rapid publication on acceptance

- support for research data, including large and complex data types

- gold Open Access which fosters wider collaboration and increased citations

- maximum visibility for your research: over $100 \mathrm{M}$ website views per year

At $\mathrm{BMC}$, research is always in progress.

Learn more biomedcentral.com/submissions 